\title{
Endotoxemia is associated with acute coronary syndrome in patients with end stage kidney disease
}

\author{
Chien-Chin $\mathrm{Hsu}^{1,2^{*}}$, Tsui-Shan Wei ${ }^{3}$, Chien-Cheng Huang ${ }^{1,4,5}$ and Yi-Ming Chen ${ }^{6}$
}

\begin{abstract}
Background: Cardiovascular disease is the major cause of death in patients with end-staged kidney disease (ESRD). Most ESRD patients have systemic inflammation, and increasing the risk of cardiovascular event. Endotoxin derived from lipopolysaccharide of Gram negative bacteria accounts for 70\% of intestinal bacteria, leading to release of proinflammatory cytokines and negative cardiovascular effect. Impaired intestinal barriers have been found in some ESRD patients, and may lead to bacteria translocation from gastrointestinal tract. We aim to investigate the association of endotoxemia in ESRD patients and acute coronary syndrome (ACS).
\end{abstract}

Methods: We collected serum from adult ESRD patients who presented to emergency department (ED) with ACS (30 patients) or without ACS (30 patients) as control from 11/01/2013 to 10/31/2014 in Chi Mei Medical Center in southern Taiwan. Clinical information and lab data were collected. We measured the endotoxin level of the serum of ESRD patients with or without ACS. We used real-time $16 \mathrm{~S}$ rDNA PCR to detect possible bacteria in the blood of the patients.

Results: The endotoxin level of ESRD patients with ACS $(0.49( \pm 0.12) \mathrm{EU} / \mathrm{mL})$ was significantly higher than that of ESRD patients without ACS $(0.1 \pm 0.08)(p<0.01)$. However, the endotoxin level was not correlated with the troponin-l level $(r=-0.12)$. Although endotoxin level was higher in ESRD patients with ACS, bacteria were not detected in the serum by using the real-time 165 rDNA PCR.

Conclusion: Endotoxin in ESRD patients with ACS was significantly higher than that without ACS. The result suggested that endotoxemia may have a contributory role to cardiovascular disease in ESRD patients.

\section{Background}

Cardiovascular disease is the major cause of death in patients with chronic kidney disease (CKD) [1]. Systemic inflammation increases the risk of cardiovascular disease in the CKD patient [2]. Most of the patients under hemodialysis therapy have systemic inflammation, which is caused in part by catheters, tubes or endotoxin existing in non-ultrapure water used for hemodialysis. However, the source of inflammation is often unknown [3]. New evidence shows that endotoxin and bacteria can be subject to intestinal translocation to the systemic blood stream

\footnotetext{
* Correspondence: nych2525@gmail.com

'Department of Emergency Medicine, Chi-Mei Medical Center, 901,

Chung-Hwa Rd, Yongkang District, Tainan 710, Taiwan

${ }^{2}$ Department of Biotechnology, Southern Taiwan University of Technology,

Tainan, Taiwan

Full list of author information is available at the end of the article
}

[4]. Endotoxin is derived from lipopolysaccharide (LPS) which is the major component of the outer membrane of Gram negative bacteria. Gram negative bacteria account for $70 \%$ of adult intestinal bacteria. Endotoxin leads to release of proinflammatory cytokines which are connected with CD 14 of immune competent cells, resulting in negative cardiovascular effect, e.g., dilatation of peripheral vessels and impaired cardiac muscle contraction [5].

Intestinal mucosa is the largest physical interface between host and microbes. Diverse microbes exist in the Intestines, consisting over 400 strains of bacteria [4]. One study showed that aerobic $\left(\sim 10^{6}\right.$ bacteria/ml $)$ and anaerobic $\left(\sim 10^{7}\right.$ bacteria/ml) bacteria were increased in the duodenum and jejunum in uremic patients; in contrast to healthy individuals, few or no bacteria existed in the area [6]. One animal study showed that uremic rats (8 of 20) were found to have bacterial translocation as 
compared to normal rats ( 1 of 20 controls) [7]. Intestinal barriers were impaired in the uremic animal [8] and CKD patients [9]. Ischemic change in the intestinal mucosa was also found in the patients undergoing hemodialysis therapy [10]. Destruction of mucosal structure increased intestinal permeability [11]. Pathological intestinal bacteria, e.g., Escherichia coli, and Salmonella typhimurium, produce several kinds of toxins. Microbial translocation in the HIV chronic infected patients has been demonstrated one of the causes in the systemic immune activation [12]. However, the role of microbial translocation in ESRD patients has not been well studied.

Several biomarkers have been used to detect and quantify microbial translocation, for example, LPS and soluble CD14 (sCD14) [13]. The sCD14 ELISA method is applicable, but it is not specific in the detection of microbial translocation, because other cytokines, e.g., INF- $\alpha$ and INF- $\beta$ may increase CD14 $[14,15]$. LPS limulus amoebocyte lysat (LAL) is currently the standard method of detecting endotoxin [16]. However, this method can only detect endotoxin produced by Gram negative bacteria, which is unable to detect microbial translocation of Gram positive bacteria in the intestine. Molecular diagnostics provides an opportunity to detect microbes, particularly viruses or bacteria which are difficult to be cultured, such as tuberculosis or anaerobic bacteria. 16S ribosomal DNA PCR is used to detect the highly-conserved prokaryotic ribosomal RNA gene. Real-time 16S PCR method after eliminating bacterial DNA in the environment can be used to quantify bacteria [17].

We aimed to evaluate the role of microbial translocation in ESRD patients with acute coronary syndrome caused by myocardial injury, contributing to elevated cardiac troponin. We measured troponin-I level, detect endotoxin level and nucleotides of microbes if any in the serum of ESRD patients by ELISA and molecular method.

\section{Methods \\ Blood samples and clinical information of ESRD patients at ED}

We collected 60 adult patients (age $>17$ years old) with ESRD presented to ED due to acute coronary syndrome $(n=30)$ or without acute coronary syndrome $(n=30)$ as control from 11/01/2013 to $10 / 31 / 2014$ in one medical center in southern Taiwan. Patients who had hospitalization due to infectious disease within 1 month, or were taking antibiotics were excluded in the study. All patients with ESRD were undergoing hemodialysis. Acute coronary syndrome was defined as clinical symptom of chest pain compatible with unstable angina, non-ST segment elevation myocardial infarction or ST-segment elevation myocardial infarction. Written informed consent for participation in the study was obtained from participants. Serum samples were collected in enotoxin-free glass tubes and stored in $-80{ }^{\circ} \mathrm{C}$. Clinical information regarding demographic feature, past history as well as lab data, cardiac sonography, electrocardiography, or coronary angiography (if any) were collected. Clinical information was de-identified. This research project was approved by Institutional Review Board of Chi Mei Medical Center (No. 10212-002).

\section{Measurement of endotoxin in serum of ESRD patients}

Serum samples were numbered and de-identified. We use the method as described previously [12] to measure endotoxin level of patients with ESRD. Serum is diluted with endotoxin-free water to $20 \%$ concentration, followed by heating to $70{ }^{\circ} \mathrm{C}$ for $10 \mathrm{~min}$ to inactivate protein in the serum. We measure and quantify lipopolysaccharide (LPS) level by Limulus Amebocyte assay (Cambrex, Verviers, Belgium) according its protocol. Briefly, a sample was mixed with the LAL supplied in the test kit and incubated at $37^{\circ} \mathrm{C}\left( \pm 1{ }^{\circ} \mathrm{C}\right)$ for $10 \mathrm{~min}$. A substrate solution is then mixed with the LAL-sample and incubated at $37^{\circ} \mathrm{C}\left( \pm 1{ }^{\circ} \mathrm{C}\right)$ for an additional $6 \mathrm{~min}$. The reaction was stopped with stop reagent. The level of endotoxin was determined by spectrophotometrically and calculated from a standard curve. The detection limit of this end point chromogenic assay was $0.01 \mathrm{EU} / \mathrm{ml}$. Samples with LPS level below the detection limit were taken as $0 \mathrm{EU} / \mathrm{ml}$. We ran samples in duplicate and subtracted the background.

\section{Real-time 16S rDNA PCR}

We use real-time 16S rDNA PCR described by Kramski, et al. [17] to quantify the possible bacteria in the blood of the patients. First, we prepare Master mix with shrimp nuclease (SNuc) treatment to eliminate bacterial double strand DNA in the environment. Cycling conditions for SNuc treatment of the master mix, $37{ }^{\circ} \mathrm{C}$ for $10 \mathrm{~min}$ followed by $72{ }^{\circ} \mathrm{C}$ for $20 \mathrm{~min}$. We use forward primer (5'-AACAGGATTAGATACCCTGGTAG-3') and reverse primer (5' GGTTCTKCGCGTTGCWTC $-3^{\prime}$ ) for PCR reaction. Cycling condition for PCR: $95{ }^{\circ} \mathrm{C}$ for $2 \mathrm{~min}$, 40 cycles of $95{ }^{\circ} \mathrm{C}$ for $2 \mathrm{~min}, 95^{\circ} \mathrm{C}$ for $5 \mathrm{~s}$, and $60{ }^{\circ} \mathrm{C}$ for $30 \mathrm{~s}$.

\section{Statistical analysis}

We use SAS software (version 9.1) to analyze categorical variables by chi-square or Fisher's exact test and continuous variables by the Wilcoxon's rank sum test.

\section{Results}

We recruited 60 ESRD patients (33 male and 27 female patients) with mean age 63.6 years who presented to emergency department (ED) from November 1, 2013 to 
October 31, 2014. Among them, 30 patients presented with ACS, and 30 patients without ACS.The later group included presentations of fever, abdominal pain, gastrointestinal tract bleeding, general malaise, and dizziness. Twenty-one patients had pre-existing coronary artery disease (CAD). Demographic and clinical information of ESRD patients presented to ED is shown in Tables 1 and 2 .

The average endotoxin level of ESRD patients with ACS was $0.49 \pm 0.12 \mathrm{EU} / \mathrm{mL}$, which was significantly higher than that of ESRD patients without ACS $(0.10 \pm 0.08$ $\mathrm{EU} / \mathrm{mL}$ ) (Table 1, Fig. 1). Systemic inflammatory marker C-reactive protein (CRP) was positively correlated with endotoxin $(r=0.574, p=0.007)$. Among those patients with ACS, there was no significant difference in endotoxin level between ESRD patients with pre-existing CAD $(0.45 \pm 0.60 \mathrm{EU} / \mathrm{mL})$ and without CAD $(0.23 \pm 0.44$ $\mathrm{EU} / \mathrm{mL}, p=0.12$ ) (Fig. 2). The average troponin-I level of ESRD patients with ACS was $1.14 \pm 3.12 \mathrm{ng} / \mathrm{mL}$, which was significantly higher than that of ESRD patients without ACS $(0.05 \pm 0.06 \mathrm{ng} / \mathrm{mL})$. However, troponin-I level was not correlated with endotoxin level $(r=-0.12)$ (Fig. 3).

Although endotoxin level was higher in ESRD patients with ACS, nucleotide sequences of bacteria were not detected in the serum by the real-time $16 \mathrm{~S}$ rDNA PCR.

\section{Discussion}

Previous study showed that endotoxin is related to systemic inflammation and atherosclerosis in CKD and peritoneal dialysis patients [18]. Chlamydial lipopolysaccharide is present in serum during acute coronary syndrome [19]. There is no study showing that endotoxin is related to ACS in ESRD patients. Our study showed that serum endotoxin level of the ESRD patients with ACS was significantly higher than that without ACS, indicating endotoxin may play an important role in ESRD patients with ACS.
One study showed that even a low endotoxin level contributes to the development of atherosclerosis [20]. Our data showed that endotoxin level of ESRD patients of ACS, regardless of pre-existing CAD, seemed to be high, however, there was no significant difference between these patients with and without pre-existing CAD $(0.45 \pm 0.60,0.23 \pm 0.44 \mathrm{EU} / \mathrm{mL}$, individually, $p=0.12)$. This can be explained by the fact that the baseline of endotoxin level in ESRD patients remains high than health individuals. Chronic status of endotoxemia contributes to chronic inflammation and the development of atherosclerosis, which might be supported by our finding that endotoxin level was positively correlated with CRP. In contrast, acute stage of cardiovascular disease, such as ACS, is associated with high level of endotoxemia, suggesting that increase in endotoxin may accelerate atherosclerosis and acute coronary syndrome.

Troponin is a good indicator in the evaluation of the risk of myocardial muscle injury or acute coronary syndrome in the early stage [21]. Although elevated troponin-I is sometimes seen in symptoms free CKD patients [22, 23], in our study, troponin-I is significantly higher in ESRD patients with ACS than that in ESRD patients without ACS; Previous study showed that Endotoxin/LPS increased directly with troponin level in patients with periodontal disease [24]. However, in our study, endotoxin is not correlated with troponin-I level in these patients. There is no dose-response relationship between endotoxin and troponin-I. It may suggest that endotoxin does not reflect the magnitude of cardiac tissue damage, and endotoxin alone cannot predict the severity of ACS. The lack of association of endotoxin with troponin -I suggests that there was minimal effect on blood clotting or platelets.

Endotoxin derived from lipopolysaccharide (LPS) has been used as a surrogate of Gram negative bacteria translocation from gastrointestinal tract. However, this method is unable to detect microbial translocation of

Table 1 Comparison of continuous variables of demographic and clinical information between ESRD patients with and without acute coronary syndrome

\begin{tabular}{llll}
\hline & ACS group $(n=30)$ & Control group $(n=30)$ & $p^{*}$ value \\
\hline Age & $65.3 \pm 11.6$ & $62.6 \pm 8.5$ & 0.68 \\
BMI $\left(\mathrm{kg} / \mathrm{m}^{2}\right)$ & $26.2 \pm 4.6$ & $25.7 \pm 3.4$ & 0.50 \\
Laboratory finding & & & \\
Prothrombin time (seconds) & $11.60(0.40)$ & $12.10(0.50)$ & 0.18 \\
INR & $1.10(0.10)$ & $1.09(0.10)$ & 0.29 \\
APTT (seconds) & $36.10(2.80)$ & $36.40(3.50)$ & 0.31 \\
Platelets $\left(\times 10^{3} / \mathrm{mm}^{3}\right)$ & $265.42(150.31)$ & $271.26(60.23)$ & 0.51 \\
CRP $(\mathrm{mg} / \mathrm{L})$ & $25.70(50.36)$ & $51.39(82.14)$ & 0.1 \\
Troponin-I $(\mathrm{ng} / \mathrm{mL})$ & $1.14(3.12)$ & $0.05(0.06)$ & $<0.01$ \\
\hline
\end{tabular}

${ }^{*} p$ vale for Wilcoxon's rank sum test 
Table 2 Comparison of categorical variables of demographic and clinical information between ESRD patients with and without acute coronary syndrome

\begin{tabular}{|c|c|c|c|}
\hline & ACS group $(n=30)$ & Control group $(n=30)$ & $p^{*}$ value \\
\hline \multicolumn{4}{|l|}{ Sex } \\
\hline Male & 18 & 14 & 0.3 \\
\hline \multicolumn{4}{|l|}{ Clinical presentations } \\
\hline Acute coronary syndrome & 30 & 0 & NA \\
\hline Fever & 0 & 11 & NA \\
\hline Abdominal pain & 0 & 7 & NA \\
\hline Gastrointestinal tract bleeding & 0 & 6 & NA \\
\hline General malaise & 0 & 4 & NA \\
\hline Dizziness & 0 & 2 & NA \\
\hline \multicolumn{4}{|l|}{ Past history } \\
\hline Coronary artery disease & 12 & 9 & 0.42 \\
\hline Congestive heart failure & 1 & 3 & 0.30 \\
\hline Diabetes & 11 & 17 & 0.12 \\
\hline Hypertension & 21 & 16 & 0.18 \\
\hline Cerebrovascular accident & 4 & 2 & 0.39 \\
\hline Malignancy & 3 & 3 & 1 \\
\hline Gastrointestinal disease $^{a}$ & 4 & 3 & 0.69 \\
\hline Recent infectious disease $\mathrm{e}^{\mathrm{b}}$ & 3 & 4 & 0.69 \\
\hline
\end{tabular}

${ }^{*} p$ value for Fisher's exact test

a Gastrointestinal disease includes hepatitis B and C, pancreatitis, peptic ulcers, and biliary tract stone

${ }^{\mathrm{b}}$ Recent infectious disease includes pneumonia, bacteremia, cellulitis, arteriovenous fistula infection

Gram positive bacteria in the intestine. We tried to search for the evidence of nucleotide sequences of any bacteria in the serum of ESRD patients. However, nucleotide sequences of bacteria were not detected in this study. It may be in part due to the methodological difficulty involved in obtaining direct samples of mesenteric lymph nodes or portal venous blood since we used serum sample to detect microbial nucleotide sequences. Alternatively, it may be explained by the result of transient bacteremia. There might be no microbes in the

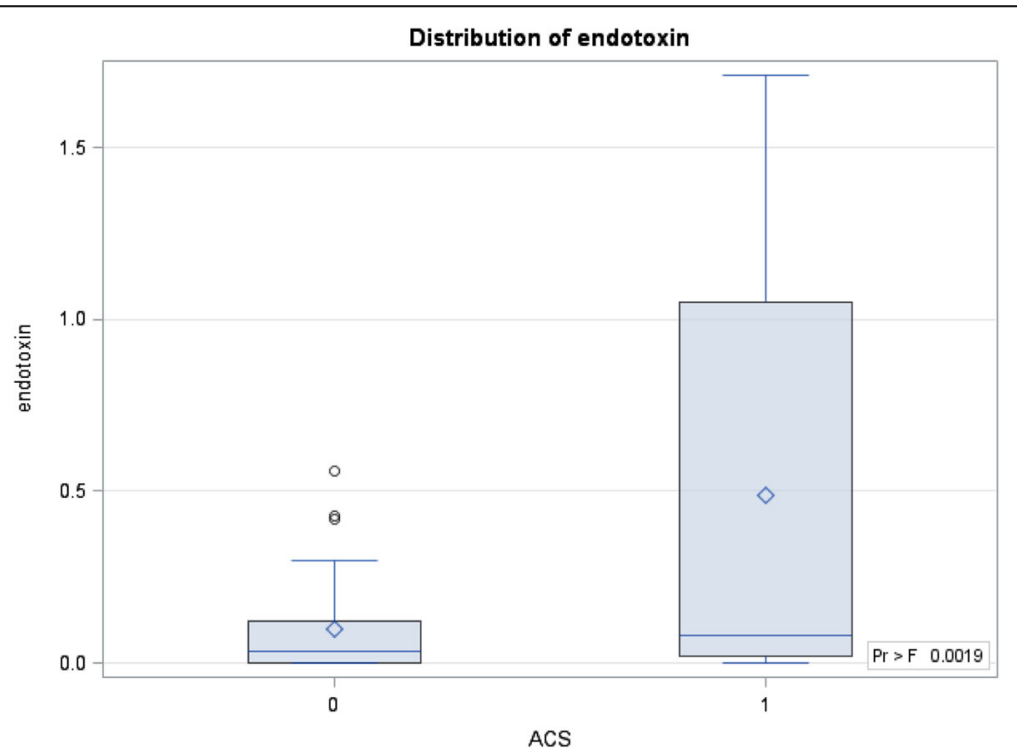

Fig. 1 Comparision of serum endotoxin level between ESRD patients with and without ACS $(p<0.01)$. The boxes indicate median, 25th, and 75th percentile; whisker caps indicate 5th and 95th percentile; open circles indicate outliers 


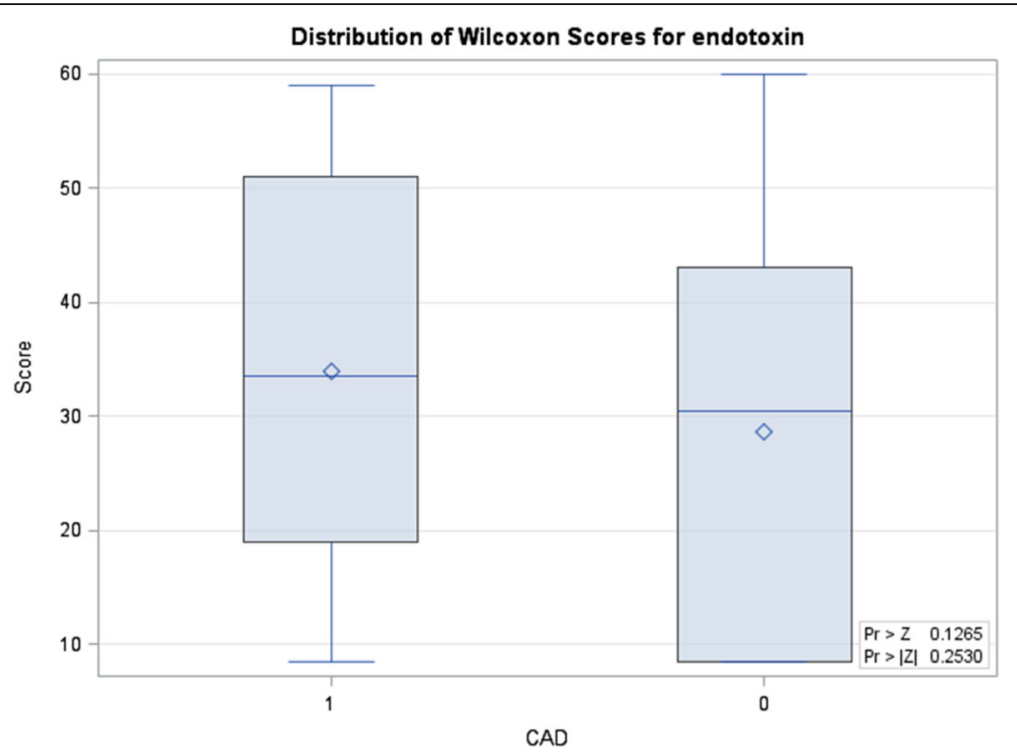

Fig. 2 Comparision of serum endotoxin level between ESRD patients with and without pre-existing coronary artery disease $(p=0.13)$. The boxes indicate median, 25th, and 75th percentile; whisker caps indicate 5th and 95th percentile; open circles indicate outliers

systemic blood stream while collecting blood of the patients. Serial blood samples might be used in the future study to improve the probability of detecting microbial nucleotides.

There were limitation in this study. We did not examine the periodontal health of the patients. Periodontal disease has been discussed as an important contributing factor to coronary artery disease. Future study for adjusting the effect of periodontal disease for CAD is needed. Secondly, we did not collect a randomized population. This makes correction for extraneous factors difficult, e.g., subclinical infection, and malnutrition.

\section{Conclusion}

Our study shows that endotoxemia is associated with ESRD patients with ACS. An elevated endotoxin is likely due to translocation of Gram negative bacteria in the gut. However, endotoxin level is not correlated with troponin-I level, indicating endotoxin itself may not reflect the magnitude of cardiac tissue damage.

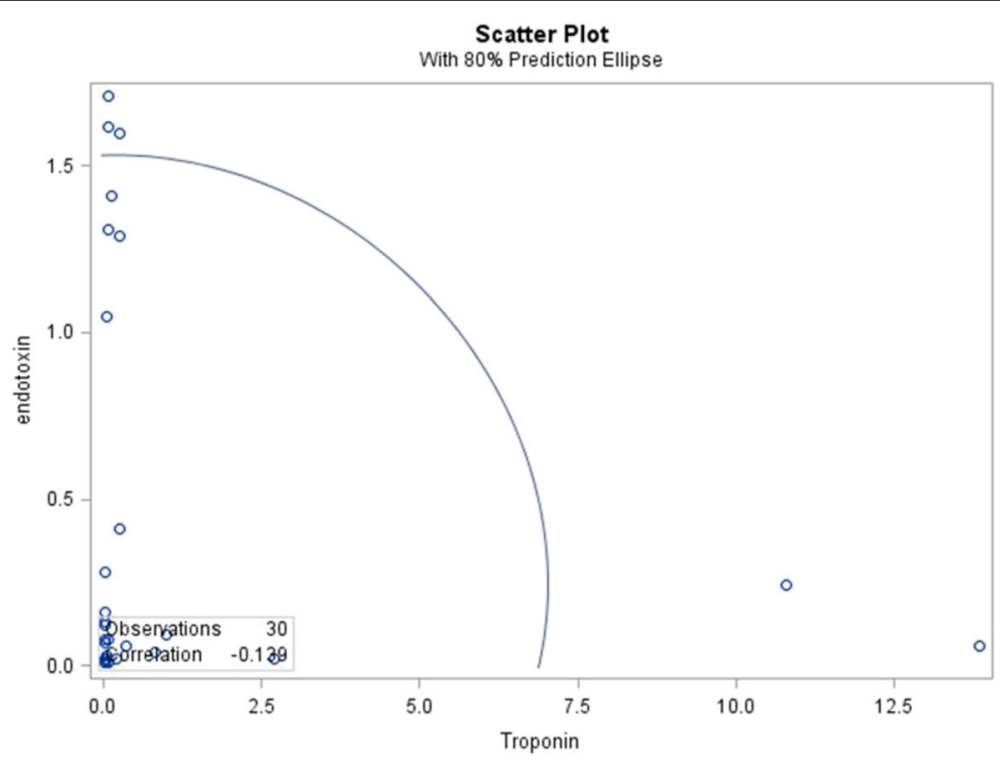

Fig. 3 Scatter plot of correlation between entotoxin and troponin-1 


\section{Abbreviations}

ACS: Acute coronary syndrome; CAD: Coronary artery disease; CKD: Chronic kidney disease; ED: Emergency department; ESRD: End-stage renal disease; LAL: Limulus amoebocyte lysat; LPS: Lipopolysaccharide; SNuc: Shrimp nuclease

\section{Acknowledgements}

The authors are grateful for all the patients who participated in this study and all the staff of the Department of Emergency Medicine, Chi-Mei Medical Center, Tainan.

\section{Funding}

The study was funded (102CM-KMU-08) by Chi-Mei Medical Center, Tainan, Taiwan.

\section{Availability of data and materials}

All data used/or analyzed in the study are available from the corresponding authors upon reasonable request.

\section{Authors' contributions}

$\mathrm{CCH}$ designed the study and drafted the manuscript. TSW carried out the measurement of endotoxin and PCR experiment. CCH collected clinical data and performed the statistical analysis and revised the manuscript. YMC contributed substantial conception of the study. All authors reviewed and approved the manuscript.

\section{Ethics approval and consent to participate}

This research project was approved by Institutional Review Board of Chi Mei Medical Center (No. 10212-002). All subjects gave written informed consent at the time of enrollment.

\section{Consent for publication}

Not applicable.

\section{Competing interests}

The authors declare that they have no competing interests.

\section{Publisher's Note}

Springer Nature remains neutral with regard to jurisdictional claims in published maps and institutional affiliations.

\section{Author details}

'Department of Emergency Medicine, Chi-Mei Medical Center, 901, Chung-Hwa Rd, Yongkang District, Tainan 710, Taiwan. ${ }^{2}$ Department of Biotechnology, Southern Taiwan University of Technology, Tainan, Taiwan. ${ }^{3}$ Department of Medical Research, Chi Mei Medical Center, Tainan, Taiwan. ${ }^{4}$ Department of Environmental and Occupational Health, College of Medicine, National Cheng Kung University, Tainan, Taiwan. ${ }^{5}$ Department of Child Care and Education, Southern Taiwan University of Science and Technology, Tainan, Taiwan. ${ }^{6}$ Department of Microbiology, College of Medicine, Kaohsiung Medical University, Kaohsiung, Taiwan.

Received: 3 November 2015 Accepted: 3 July 2017

Published online: 12 July 2017

\section{References}

1. Go AS, et al. Chronic kidney disease and the risks of death, cardiovascular events, and hospitalization. N Engl J Med. 2004;351(13):1296-305.

2. Wanner C, Drechsler C, Krane V. C-reactive protein and uremia. Semin Dial. 2009;22(4):438-41.

3. Yao Q, et al. Systemic inflammation in dialysis patients with end-stage renal disease: causes and consequences. Minerva Urol Nefrol. 2004;56(3):237-48.

4. Kotanko P, Carter M, Levin NW. Intestinal bacterial microflora-a potential source of chronic inflammation in patients with chronic kidney disease. Nephrol Dial Transplant. 2006;21(8):2057-60

5. Kumar A, Haery C, Parrillo JE. Myocardial dysfunction in septic shock. Crit Care Clin. 2000;16(2):251-87.

6. Simenhoff ML, et al. Bacterial populations of the small intestine in uremia. Nephron. 1978;22(1-3):63-8.

7. de Almeida Duarte JB, et al. Bacterial translocation in experimental uremia. Urol Res. 2004;32(4):266-70.
8. Magnusson M, et al. Increased intestinal permeability to differently sized polyethylene glycols in uremic rats: effects of low- and high-protein diets. Nephron. 1990;56(3):306-11.

9. Magnusson $\mathrm{M}$, et al. Impaired intestinal barrier function measured by differently sized polyethylene glycols in patients with chronic renal failure. Gut. 1991;32(7):754-9.

10. Diebel $L$, et al. Gastric intramucosal acidosis in patients with chronic kidney failure. Surgery. 1993;113(5):520-6.

11. Khanna $A$, et al. Intestinal and hemodynamic impairment following mesenteric ischemia/reperfusion. J Surg Res. 2001;99(1):114-9.

12. Brenchley JM, et al. Microbial translocation is a cause of systemic immune activation in chronic HIV infection. Nat Med. 2006;12(12):1365-71.

13. Rajasuriar $\mathrm{R}$, et al. Biological determinants of immune reconstitution in HIV-infected patients receiving antiretroviral therapy: the role of interleukin 7 and interleukin 7 receptor alpha and microbial translocation. J Infect Dis. 2010;202(8):1254-64.

14. Brettschneider J, et al. The macrophage activity marker SCD14 is increased in patients with multiple sclerosis and upregulated by interferon beta-1b. J Neuroimmunol. 2002;133(1-2):193-7.

15. Carotenuto $P$, et al. Antiviral treatment with alpha interferon up-regulates CD14 on liver macrophages and its soluble form in patients with chronic hepatitis B. Antimicrob Agents Chemother. 2005:49(2):590-9.

16. Hurley JC. Endotoxemia: methods of detection and clinical correlates. Clin Microbiol Rev. 1995:8(2):268-92.

17. Kramski M, et al. Novel sensitive real-time PCR for quantification of bacterial $16 S$ rRNA genes in plasma of HIV-infected patients as a marker for microbial translocation. J Clin Microbiol. 2011;49(10):3691-3.

18. Szeto CC, et al. Endotoxemia is associated with better clinical outcome in incident Chinese peritoneal dialysis patients: a prospective cohort study. Perit Dial Int. 2010;30(2):178-86.

19. Tiirola T, et al. Chlamydial lipopolysaccharide is present in serum during acute coronary syndrome and correlates with CRP levels. Atherosclerosis. 2007:194(2):403-7.

20. Wiedermann CJ, et al. Association of endotoxemia with carotid atherosclerosis and cardiovascular disease: prospective results from the Bruneck Study. J Am Coll Cardiol. 1999;34(7):1975-81.

21. Alpert JS, et al. Myocardial infarction redefined-a consensus document of The Joint European Society of Cardiology/American College of Cardiology Committee for the redefinition of myocardial infarction. J Am Coll Cardiol. 2000;36(3):959-69.

22. Freda BJ, et al. Cardiac troponins in renal insufficiency: review and clinical implications. J Am Coll Cardiol. 2002;40(12):2065-71.

23. Chen $\mathrm{S}$, et al. Cardiac troponin I in non- acute coronary syndrome patients with chronic kidney disease. PLOS One. 2013:8(12):e82752.

24. Goteiner D, et al. Endotoxin levels are associated with high-density lipoprotein, triglycerides, and troponin in patients with acute coronary syndrome and angina: possible contributions from periodontal sources. J Periodontol. 2008:79(12):2331-9.

\section{Submit your next manuscript to BioMed Central and we will help you at every step:}

- We accept pre-submission inquiries

- Our selector tool helps you to find the most relevant journal

- We provide round the clock customer support

- Convenient online submission

- Thorough peer review

- Inclusion in PubMed and all major indexing services

- Maximum visibility for your research

Submit your manuscript at www.biomedcentral.com/submit 